Journal of Animal and Veterinary Advances 11 (18): 3312-3317, 2012

ISSN: $1680-5593$

(C) Medwell Journals, 2012

\title{
Expression of CSFV E2 Protein in Eukaryotic Cells and Characterization of Immunity in Animals
}

\author{
${ }^{1,2}$ Jian-Ling Liu, ${ }^{1}$ Kang-Kang Guo, ${ }^{1}$ Zheng-Yuan Su, ${ }^{1}$ Xin-Gang Xu, \\ ${ }^{1} \mathrm{He}-\mathrm{Lin} \mathrm{Li},{ }^{1}$ Peng-Bo Ning, ${ }^{1} \mathrm{Hai}-\mathrm{Xia}$ Hong, ${ }^{1}$ Xiao-Yun Yang and ${ }^{1}$ Yan-Ming Zhang \\ ${ }^{1}$ College of Veterinary Medicine, Northwest A\&F University, \\ Yangling, 712100 Shaanxi, P.R. China \\ ${ }^{2}$ College of Life Science, Northwest University, Xi' an, 710069 Shaanxi, P.R. China
}

\begin{abstract}
The viral envelope glycoprotein E2 is major target for eliciting protective antibodies against CSFV in infected animals. To express CSFV E2 protein on eukaryotic cells, the recombinant retroviral vector pBabepuro-E2 was constructed by inserting full-length CSFV Shimen strain E2 region into pBabe-puro. Both of the recombinant retroviral vector and pVSVg plasmid were transfected eukaryotic 293GP cells using method of calcium phosphate coprecipitation in where thus the recombinant pseudovirus were packaged and propagated. The PK-15 cells were infected with recombinant pseudovirus. The recombinant PK-15 cells were screened by spytomycin resistance and the expression of E2 protein was detected by Flow Cytometer (FCM). The activity of recombinant $\mathrm{E} 2$ protein to induce immune responses was evaluated in Balb/c mice and unvaccinated pigs. The results showed that CSFV E2 protein was successfully expressed on PK-15 cells membrane. Anti-E2 antibody induced in experimental animals was detected by Enzyme Linked Immunosorbent Assay (ELISA). Moreover, the virus challenge indicated that the immunized pigs generated effective protection against virulent CSFV. These results indicated that a retroviral-based E2-vaccine is able to induce high level of specific antibodies and exhibits similar protective capability with that induced by the C-strain.
\end{abstract}

Key words: Classical Swine fever virus, E2 protein, retroviral vector, eukaryotic expression, immune protection experiment

\section{INTRODUCTION}

Classical Swine Fever (CSF), caused by Classical Swine Fever Virus (CSFV) is a highly infectious hemorrhagic disease of pigs. It is an important infectious disease that must be reported to Office International des Epizooties (OIE) and considered the most damaging porcine disease all around the world (Greiser-Wilke et al., 2007). CSFV belongs to the genus Pestivinus within the family Flaviviridae, it is a small enveloped virus encompassing a single-stranded positive RNA of about $12.3 \mathrm{~kb}$ (Ribbens et al., 2004). Its genome contains a single large Open Reading Frame (ORF) encoding a polyprotein of 4,000 amino acids which is processed by cellular and viral proteases into 12 mature viral proteins (Horzinek, 1991). The virion consists of four structural proteins, the core protein and the glycoproteins E0, E1 and E2 among which E2 that encoded protein gp55 is the major protective antigenic protein. Its full-length is about $1.2 \mathrm{~kb}$, encoding 380 amino acid residues (Rumenapf et al., 1989; Hulst et al., 1993; Xu and Liu, 2008).
Because of the convenient packaging and the ability to integrate into chromosomal DNA in nucleus, ensuring its long-term persistence and stable transmission to the future transduced cells, the retroviral vector has become a common delivery vehicle for eukaryotic cells (Weiss and Chakraborty, 2001; Cherry et al., 2000). In this study, researchers integrated the CSFV E2 region into PK- 15 cells genome via a retroviral vector (pBabe-puro) and E2 protein was expressed in $\mathrm{PK}-15$ cells cultured in vitro, detected by Flow CytoMeter (FCM). The expressed E2 protein on recombiant $\mathrm{PK}-15$ cells was used to immunize $\mathrm{Balb} / \mathrm{c}$ mice by intraperitoneal injection and unvaccinated pigs by intramuscular injection. The experimental animals generated anti-E2 antibody that was confirmed by Enzyme Linked Immunosorbent Assay (ELISA). Pigs immunized with recombinant PK-15 cells culture did not clinic signs and no pathologic findings were observed for pathologic examination when all immunized pigs were each challenged with the highly virulent CSFV Shimen strain, its indicated that the recombinant PK-15 cells could

Corresponding Author: Yan-Ming Zhang, College of Veterinary Medicine, Northwest A\&F University, Yangling, 712100 Shaanxi, P.R. China 
provided solid protection against CSFV. The study showed that the recombiant PK-15 cells is potential as vaccine candidate against CSF in pigs.

\section{MATERIALS AND METHODS}

Reagents: Horseradish Peroxidase (HRP) conjugated rabbit anti-pig $\operatorname{IgG}$ was purchased from Zhongshan Bioengineering company, China; FITG conjugated rabbit anti-pig IgG was from Sigma; PRMI-1640 medium was from Gibco; McAb purified swine fever ELISA antigen, positive serum, negative serum and enzyme conjugated secondary antibody (rabbit anti-pig IgG) were from Control Institute of Veterinary Bioproducts and Pharmaceuticals, China; Restriction enzeymes, T4 DNA ligase and dNTP were respectively purchased from Promega corp, Gibco corp and Hua-Mei Biotechnology company, China.

Vectors and cells: pUC119-E2 plasmid, pBabe-puro retroviral vector, pVSVg vector, 293 GP cells, PK-1 5 cells and $E$. coli $\mathrm{DH} 5 \alpha$ strain were obtained from the preservation of the lab. pUC119-E2 contains the E2 region of CSFV Shimen strain. The pBabe-puro retroviral vector has 6 unique restriction sites and carries an ampicillin-resistant gene and a spytomycin-resistant gene (Vile et al., 1996). The pVSVg vector expresses the G protein of Vesicular Stomatitis Virus (VSV) under the control of the cytomegalovirus immediate early promoter (Morgenstern and Land, 1990; Yee et al., 1994). Cells were grown in Dulbecco's Modified Eagle's Medium (DMEM) supplemented with $10 \%$ fetal calf serum.

Construction and identification of recombinant retroviral vector: A pair of primers were synthesized according to reported sequence of CSFV E2. E2-F: 5'-GGATCCATGGG TTGCTCTTTCTCTATC TTCC-3'. E2-R: 5'-GAATTCTTA CGCCTCCGCTTGGGATATGAG- 3 '. The $1.2 \mathrm{~kb}$ full-length E2 gene of CSFV Shimen strain was amplified by PCR with E2-F and E2-R from plasmid pUC119-E2 and inserted into pBabe-puro retroviral vector between BamH and EcoR sites, giving rise to pBabe-puro-E2. This recombinant plasmid was transfected into $E$. coli $\mathrm{DH} 5 \alpha$ cells, the positive cellular clones were screened by ampicillinresistant LB and identified by restriction digestion with restriction enzymes BamHIand EcoRI. Two fragments of 5.1 and $1.2 \mathrm{~kb}$ were obtained just as the length of pBabe-puro and E2 fragment which the insert position, size and reading frame were right by restriction digestion and sequence analysis.

Packaging of recombinant pseudovirions: The resulting recombiant plasmid (pBabe-puro-E2) and pVSVg vector were cotransfected into $293 \mathrm{GP}$ cells using methods of calcium phosphate coprecipitation (Jordan and Wurm, 2004) and the recombinant pseudovirions were packaged and propagated. The $36-48 \mathrm{~h}$ post-cotranfected, culture medium were collected, centrifuged thus the supernatant containing the recombinant virions (pseudovirus) were obtained.

Infection of PK-15 cells with recombinant pseudovirus: PK-15 cells were passaged to culturing flasks in a low density $12 \mathrm{~h}$ before infection at first. The culture medium of PK-15 cells was abandoned and added the supernatant obtaining recombiant virions (pseudovirus) mixed with polybrene in a concentration of $8 \mu \mathrm{g} \mathrm{mL} \mathrm{m}^{-1}$ in it. The 12-24 h post-infected, the rest pseudovirus were removed by replacing medium. The $48 \mathrm{~h}$ post-infected, the spytomycin was added with a concentration of $5 \mu \mathrm{g} \mathrm{mL}^{-1}$. In this test, about $20 \%$ PK-15 cells infected by the recombiant pseudovirus survived due to obtaining the resistance to spytomycin while all the cells of the negative control were killed. The positive cellular clones were destination recombiant cells that would expressed E2 protein.

E2 protein detection: About 36-48 $\mathrm{h}$ post-added spytomycin, the surviving pseudovirus infected PK-15 cells were collected, lysed by freeze thawing for three times followed by sonication. Protein was resolved by electrophoresis in a $12 \%$ Sodium Dodecyl SulfatePolyacrylamide Gel Electrophoresis (SDS-PAGE) and then transferred onto a pyroxylin membrane. Proteins were blocked overnight at $4^{\circ} \mathrm{C}$ with $5 \%$ Bovine Serum Albumin (BSA) and then probed with anti-CSFV sera (1:1000 dilution). After extensive washes in $0.05 \%$ Tween- 20 in phosphate-buffered saline, a secondary goat anti-pig sera conjugated to Horseradish Peroxidase (1:3000 dilution) were applied to the blots for $1 \mathrm{~h}$ at room temperature. Washes were repeated for three times and the target proteins were detected by DAB chromogenic substrate staining. At this tests the negative control PK-15 cells were treated by the same methods.

The expression of E2 proteins on the resulting cell membrane were detected by FCM. The collected surviving pseudovirus infected PK-15 cells (positive cells) were bounded with pig anti-CSFV sera and then stained with rabbit anti-pig secondary IgG conjugated to FITC. Blank PK-15 cells and recombinant cells were analyzed by FCM synchronously.

Immunization of mice: Six, 6-8 weeks old female Balb/c mice were divided into 2 groups randomly. Group $1(n=4)$ mice were immunized with $1 \times 10^{7}$ cells $/ 0.5 \mathrm{~mL}$ of recombinant PK-15 cells by intraperitoneal injection and 
boosted twice at 2 weeks intervals. Group $2(n=2)$ mice were immunized with the blank (uninfected) PK-15 cells using the same dose and procedure.

Immunization pigs: Twenty one, 12 weeks old healthy crossbred pigs purchased from a CSF-free farm were tested CSF negative to evaluate the efficacy of the recombinant PK-15 cells. The pigs were assigned to 3 groups. Group $1(\mathrm{n}=9)$ pigs were immunized with approximately $1 \times 10^{7}$ of recombinant PK-15 cells and Group $3(n=6)$ pigs were immunized with approximately $1 \times 10^{7}$ of blank PK-15 cells by intramuscular injection followed by once times of boost at 2 weeks intervals. Group $2(\mathrm{n}=6)$ pigs were immunized with $200 \mathrm{RID}_{50}(50 \%$ Rabbit Infection Dose) of the Chinese lapinized vaccine (C-strain) by the same immunization procedure with Group 1 and 3.

Antibody detection: Serum samples of immunized mice were collected at 6 weeks after first immunization and serum samples of immunized pigs were collected every week after 1 weeks at the second immunization for 7 times. All sera were subjected to detection by Enzyme Linked Immunosorbent Assay (ELISA) and tested in duplicate. Optical Density at $490 \mathrm{~nm}\left(\mathrm{OD}_{490}\right)$ was read with a ELISA reader. Seroconversion was considered to be positive if the $O D_{490}$ value $\geq 0.2$ otherwise to be negative.

Virulent challenge: About 7 weeks following the last immunization, all the immunized pigs were each challenged intramuscularly with $1 \mathrm{~mL}$ blood stock of the highly virulent CSFV Shimen strain freshly rejuvenated in pigs. Daily rectal temperature and clinical signs of disease were recorded. Pigs that were severely affected (assessed by fever, apastia, astasia, diarrhoea and flushing of the skin). The pigs with obvious clinic signs of CSF were euthanized and subjected to general pathological examinations. Surviving pigs were killed at day 14 after challenge.

\section{RESULTS}

Expression of E2 protein on PK-15 cells: The positive PK-15 cell cultures were lysed $20 \mathrm{~h}$ post-infected by recombiant pseudovirus and the extracted products were separated by SDS-PAGE and detected by Western blot (Fig. 1) for confirming the expression of the $\mathrm{E} 2$ protein by recombinant cells. The results indicated that the recombinant $\mathrm{PK}-15$ cells expressed $\mathrm{E} 2$ protein while the blank PK-15 cells did not yield corresponding protein. And the expression of E2 protein by the recombinant
PK-15 cells was further tested by FCM analysis. Two different peaks were formed (Fig. 2), the green curve representing the recombinant $\mathrm{PK}-15$ cells and the red curve representing the blank PK-15 cells (control cells). The results demonstrated that CSFV E2 protein was expressed successfully in recombinant $\mathrm{PK}-15$ cells.

Recombinant PK-15 cells elicited CSFV-E2-specific antibodies in immunized animals: The CSFV-E2-specific antibodies in mice were detected by ELISA. The $\mathrm{OD}_{490}$ value represented the antibodies titers in sera, the CSFV-

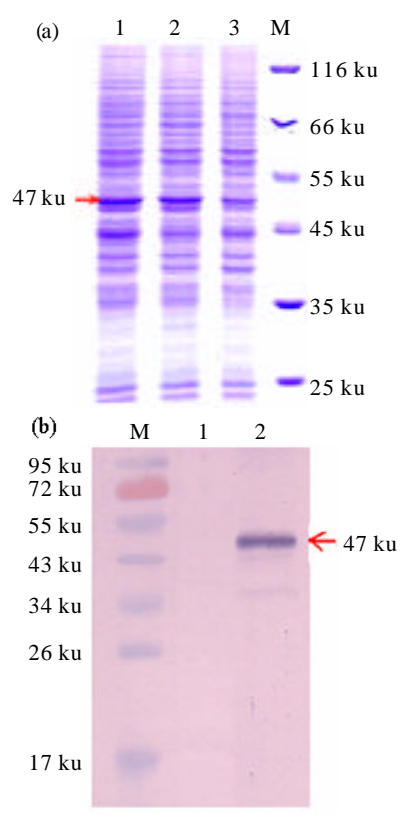

Fig. 1: Expression of E2 protein in recombinant PK-15 cells detected by SDS-PAGE and Western blot. a) SDS-PAGE of E2 protein expressed on $\mathrm{PK}-15$ cells. Lane 1-3: The pseudovirus infected PK-15 cells; Lane M: Prestained protein marker; b) Western blot of E2 protein expressed on PK-15 cells. Lane 1: Negative control PK-15 cells; Lane 2: The pseudovirus infected PK-15 cells expressing E2 protein

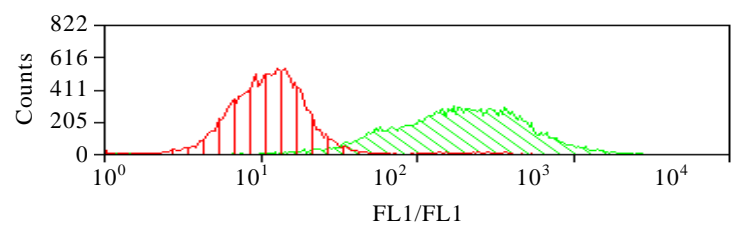

Fig. 2: Detection results of E2 protein expressed on PK-15 cells by FCM. The red peak representing the blank PK-15 cells and the green peak representing the recombinant $\mathrm{PK}-15$ cells 
Table 1: Detection of CSFV-E2-specific antibodies induced by recombinant PK-15 cells of pigs Weeks of second post-immunization (mean $\pm \mathrm{SD}$ )

\begin{tabular}{|c|c|c|c|c|c|c|c|}
\hline Items & 1 & 2 & 3 & 4 & 5 & 6 & 7 \\
\hline Group 1 & $0.59 \pm 0.032$ & $0.61 \pm 0.013$ & $0.63 \pm 0.014$ & $0.63 \pm 0.026$ & $0.62 \pm 0.017$ & $0.64 \pm 0.030$ & $0.62 \pm 0.018$ \\
\hline Group 2 & $0.58 \pm 0.023$ & $0.62 \pm 0.031$ & $0.65 \pm 0.019$ & $0.65 \pm 0.015$ & $0.63 \pm 0.020$ & $0.62 \pm 0.018$ & $0.63 \pm 0.014$ \\
\hline Control & $0.08 \pm 0.013$ & $0.07 \pm 0.021$ & $0.09 \pm 0.017$ & $0.07 \pm 0.022$ & $0.07 \pm 0.016$ & $0.08 \pm 0.011$ & $0.06 \pm 0.023$ \\
\hline
\end{tabular}

E2-specific antibodies of serum samples were positive if the $\mathrm{OD}_{490}$ values $\geq 0.2$. The $\mathrm{OD}_{490}$ values of the 4 immunized mice were $0.45,0.47,0.46$ and 0.43 , respectively. In contrast, the $\mathrm{OD}_{490}$ values of negative control mice which were injected with blank PK-15 cells were 0.05 and 0.07 , respectively. The standard positive sera had an $O D_{490}$ value of 0.69 and the standard negative sera had an $\mathrm{OD}_{490}$ value of 0.07 . The results indicated that the CSFV-E2-specific antibodies were induced in mice by immunization with recombinant PK-15 cells. Furthermore, The CSFV-E2-specific antibodies in pigs were measured by ELISA. The $\mathrm{OD}_{490}$ values were seen in Table 1 . The standard anti-CSFV positive serum had an $\mathrm{OD}_{490}$ value of $0.75 \pm 0.12$ and the standard negative serum of $0.07 \pm 0.02$, it showed that the detection of ELISA was tenable. The average $\mathrm{OD}_{490}$ values of recombinant $\mathrm{PK}-15$ cells immunized group (Group 1), Chinese lapinized vaccine (C-strain) immunized group (Group 2) and blank PK-15 cells immunized group (Group 3) were $0.620 \pm 0.031$, $0.626 \pm 0.022$ and $0.07 \pm 0.013$, respectively. The results showed that the recombinant $\mathrm{PK}-15$ cells induced CSFV-E2-specific antibodies in pigs successfully.

Pigs immunized with recombinant PK-15 cells were protected from lethal challeng with virulent CSFV: The clinical symptoms and pathological changes of pigs were observed following virulent CSFV challenge. The pigs immunized with recombinant PK-15 cells (Group 1) and Chinese lapinized vaccine (C-strain) (Group 2) developed obvious clinical signs. All surviving pigs were euthanized for pathological examination on 14 day post-challenge. At necropsy, no pathological findings were observed for pigs immunized with recombinant PK-15 cells or Chinese lapinized vaccine.

In contrast, the control pigs immunized with blank PK-15 cells immunized pigs exhibited a severe clinical symptoms typical of CSF such as pyrexia (rectal temperature $40.5-42^{\circ} \mathrm{C}$ ), depression, anorexia, respiratory distress, tremor, diarrhoea, skin petechiae and ecchymosis of the ears, abdomen, perineum and the inner side of the limb skin, paralysis of hind legs and nervous symptom in the late stage of challenge.

Two pigs succumbed to disease on 7 Day PostChallenge (DPC) and others were euthanized in a moribund state to avoid suffering on 14 DPC. Post-mortem examination of control pigs revealed pathological lesions typical of virulent CSFV infection such as tonsil necroses, marginal spleen infarcts, ileocecal valve ulcers, lymph hemorrhages and edemas, urinary bladder hemorrhages and petechiae and kidney petechiae.

\section{DISCUSSION}

In recent years, genetically engineered vaccine strategies have been studied and used in some fields such as medicine, veterinary medicine and livestock farm (Arnon and Ben-Yedidia, 2003). And using an expression system to generate the complete CSFV E2 protein which contains the major antigenic determinants has become one of the most important methods to produce CSFV vaccine. Prokaryotic expression systems are capable of generating highly yield of protein however, this protein lack the post-translational modifications including regulation folding, solubility and biological activity (Ju et al., 2005). In order to obtain entire biological activity E2 protein, post-translational modifications including formation of disulfide bond and oligomer, glycosylation, phosphorylation and degradation of protease are necessary. Using a eukaryotic expression system can overcome these difficulties. Some studies have reported that recombinant virus-vector vaccines with the $E 2$ gene of CSFV provided protective immunity (Rumenapf et al., 1991; De Smit et al., 2001a, b; Lin et al., 2012).

Recombinant retroviral pseudovirus which integrated E2 region expressing the major E2 structural protein of CSFV and the pVSVg vector which expressed the G protein of vesicular stomatitis virus were coprecipitated transfected 293GP cells. The recombinant pseudovirus were amplified by infecting PK-15 cells. For E2 region contained a signal peptide which could mediate the protein to transfer, E2 protein was stably expressed on the cell membrane. In this study, researchers took the advantages of eukaryotic expression system in which the expressed protein was modified by glycosylation and phosphorylation so as to generate full protective antigen. In addition, the PK-15 cells were derived from pig thus the recombinant cells could not induced anti-PK-15 antibody, avoiding expensive cumbersome procedure of protein 
purification. The highly yield of protein and efficient post-translational modification upgrade the immunogenicity and protection of CSFV vaccine, laying a foundation on the application study of CSFV genetically engineered vaccine.

After spytomycin screening, part of the pseudovirus infected PK-15 cells were integrated by the retrovirus gene to obtain resistance to spytomycin. It was demonstrated from Western blot and FCM detection that E2 protein was successfully expressed on the PK-1 5 cells membrane. The CSFV-E2-specific antibodies in sera of immunized mice and pigs were highly titers. Moreover, the PK-15 cells were easily cultured in vitro (Rumenapf $e t$ al., 1991), ensuring that sufficient expression of $\mathrm{E} 2$ protein. Thus, it is an effective way of preparing CSFV recombinant virus-vector vaccines.

\section{CONCLUSION}

Virulent challenge demonstrated that the pigs immunized with recombinant PK-15 cells induced antiCSFV antibodies conferred full protection against a severe virulent CSFV challenge. The tests indicated that the recombinant PK-15 cells would become a new CSFV vaccine and play an important role in preventing and controlling the disease of CSF. Pigs immunized by Chinese lapinized vaccine also generated highly yield of antibody and provided complete protection against virulent CSFV challenge, it demonstrated the Chinese lapinized vaccine was an effective vaccine in preventing and controlling the disease of CSF. Compared the recombinant immunized pigs produced slightly less amount of antibody comparing to the pigs immunized with Chinese lapinized vaccine. Generally, because of the continuous proliferation, the attenuated vaccine induces higher titer of antibody and the immunity lasts longer than that of cell vaccine (De Smit et al., 2001b). Thus, it gives us a clue in the further study to improve the expression effectiveness of the CSFV subunit vaccines.

\section{ACKNOWLEDGEMENTS}

The research was funded by Shaanxi province 13115 major scientific and technological special project (2010ZDKG-71). Researchers are grateful to those who participated in this study via technical assistance, animal experimentation and critical reading of the manuscript. Jian-Ling Liu and Kang-Kang Guo contributed equally to this research.

\section{REFERENCES}

Arnon, R. and T. Ben-Yedidia, 2003. Old and new vaccine approaches. Int. Immunopharmacol., 3: 1195-1204.

Cherry, S.R., D. Biniszkiewicz, L. van Parijs, D. Baltimore and R. Jaenisch, 2000. Retroviral expression in embryonic stem cells and hematopoietic stem cells. Mol. Cell Biol., 20: 7419-7426.

De Smit, A.J., A. Bouma, E.P. de Kluijver, C. Terpstra and R.J.M. Moormann, 2001a. Duration of the protection of an E2 subunit marker vaccine against classical swine fever after a single vaccination. Vet. Microbiol., 78: 307-317.

De Smit, A.J., A. Bouma, H.G.P. van Gennip, E.P. de Kluijver and R.J.M. Moormann, 2001b. Chimeric (marker) c-strain viruses induce clinical protection against virulent Classical Swine Fever Virus (CSFV) and reduce transmission of CSFV between vaccinated pigs. Vaccine, 19: 1467-1476.

Greiser-Wilke, I., S. Blome and V. Moennig, 2007. Diagnostic methods for detection of Classical swine fever virus status quo and new developments. Vaccine, 25: 5524-5530.

Horzinek, M.C., 1991. Pestiviruses-taxonomic perspectives. Arch. Virol. Suppl., 3: 1-5.

Hulst, M.M., D.F. Westra, G. Wensvoort and R.J. Moormann, 1993. Glycoprotein el of hog cholera virus expressed in insect cells protects swine from hog cholera. J. Virol., 67: 5435-5442.

Jordan, M. and F. Wurm, 2004. Transfection of adherent and suspended cells by calcium phosphate. Methods, 33: 136-143.

Ju, W., J. Liu, W. Xiao, M. Liu and X. Qu, 2005. Construction of a eukaryotic expression system of hsp65 gene from Mycobacterium tuberculosis and anti-hsp65 igg produced in mice. J. Med. Microbiol., 54: 3-6.

Lin, G.J., M.C. Deng, Z.W. Chen, T.Y. Liu and C.W. Wu et al., 2012. Yeast expressed classical swine fever e2 subunit vaccine candidate provides complete protection against lethal challenge infection and prevents horizontal virus transmission. Vaccine, 30: 2336-2341.

Morgenstern, J.P. and H. Land, 1990. Advanced mammalian gene transfer: High titre retroviral vectors with multiple drug selection markers and a complementary helper-free packaging cell line. Nucleic Acids Res., 18: 3587-3596.

Ribbens, S., J. Dewulf, F. Koenen, H. Laevens and A. De Kruif, 2004. Transmission of classical swine fever: A review. Vet. Q., 26: 146-155. 
Rumenapf, T., G. Meyers, R. Stark and H.J. Thiel, 1989. Hog cholera virus-characterization of specific antiserum and identification of cdna clones. Virology, 171: 18-27.

Rumenapf, T., R. Stark, G. Meyers and H.J. Thiel, 1991. Structural proteins of hog cholera virus expressed by vaccinia virus: Further characterization and induction of protective immunity. J. Virol., 65: 589-597.

Vile, R.G., A. Tuszynski and S. Castleden, 1996. Retroviral vectors. From laboratory tools to molecular medicine. Mol. Biotechnol., 5: 139-158.
Weiss, S. and T. Chakraborty, 2001. Transfer of eukaryotic expression plasmids to mammalian host cells by bacterial carriers. Curr. Opin. Biotechnol., 12: $467-472$.

$\mathrm{Xu}, \mathrm{X}$.G. and H.J. Liu, 2008. Baculovirus surface display of E2 envelope glycoprotein of classical swine fever virus and immunogenicity of the displayed proteins in mouse model. Vaccine, 26: 5455-5460.

Yee, J.K., A. Miyanohara, P. LaPorte, K. Bouic, J.C. Burns and T. Friedmann, 1994. A general method for the generation of high-titer, pantropic retroviral vectors: Highly efficient infection of primary hepatocytes. Proc. Nat. Acad. Sci. USA., 91: 9564-9568. 